\title{
La dimensión ambiental del desarrollo sostenible en el contrato estatal de obra ${ }^{1}$
}

\author{
The environmental dimension of sustainable \\ development in the state construction contract
}

Angélica Higuera Rodriguez*

\section{Resumen}

El contrato estatal de obra posee un carácter instrumental como herramienta de política pública para concretar las metas y objetivos formulados por el Estado con arreglo a lo dispuesto en el artículo 2 de la Constitución Política de 1991 y el artículo 3 de la Ley 80 de 1993. En esta contratación los particulares interactúan como destinatarios y colaboradores en el cumplimiento de los propósitos que se buscan con las políticas y esta interacción, imprime un importante dinamismo a los procesos de crecimiento económico y social del país. Sin embargo, la construcción de obras públicas resulta una de las actividades que más impacto genera en el consumo de recursos naturales, en la contaminación y la producción de residuos; aunado a ello, en la actualidad se ha visto un aumento en el deterioro ambiental, principalmente por los procesos de cambio climático y la pérdida

1 Artículo de investigación.

Abogada de la Universidad Santo Tomás, sede Bogotá; especialista en Derecho Ambiental de la Universidad del Rosario y magister en Derecho Contractual Público y Privado de la Universidad Santo Tomás, sede Bogotá. 
de la integridad de la biosfera. Bajo este escenario, este escrito hace una reflexión sobre los principales elementos con que se puede planificar y gestionar el ambiente en el contrato de obra pública, con el propósito de determinar si el carácter instrumental de este tipo de contrato se articula o no con la aplicación del principio de desarrollo sostenible consagrado en la Constitución Política de 1991 y en la Ley 99 de 1993, desde su dimensión ambiental.

\section{Palabras clave}

Desarrollo sostenible, contrato estatal de obra, política pública, gestión y planificación ambiental.

\section{Abstract}

The state construction contract has an instrumental nature as a public policy tool to specify the goals and objectives formulated by the State in accordance with the provisions of article 2 of the 1991 Political Constitution and article 3 of Law 80 of 1993. In this contracting, individuals interact as recipients and collaborators in the fulfillment of the purposes that are sought with the policies and this interaction, prints an important dynamism to the processes of economic and social growth in the country. However, the construction of public works is one of the activities that generates the greatest impact on the consumption of natural resources, on pollution and the production of waste, in addition to this, at present there has been an increase in environmental deterioration mainly by the processes of climate change and the loss of the integrity of the biosphere. Under this scenario, this writing makes a reflection on the main elements with which the environment can be planned and managed in the public works contract, with the purpose of determining whether the instrumental nature of this type of contract is articulated or not with the application of the principle of sustainable development ens- 
hrined in the Political Constitution of 1991 and in Law 99 of 1993, from its environmental dimension.

\section{Key words}

Sustainable development, state construction contract, public policy, environmental management and planning.

\section{Introducción}

La construcción de obras tiene un impacto significativo dentro de la contratación pública, por ser una herramienta estratégica del país en términos de crecimiento social y económico. Lo anterior se explica porque esta actividad es un medio para alcanzar los objetivos estatales previstos en el artículo 2 de la Constitución Política de 1991, y como quiera que esté al servicio de los intereses generales, es una manifestación de la función administrativa prevista en el artículo 209 de la Constitución Política de 1991 (Aponte, 2014). Desde una visión de la nueva gestión pública, este tipo de contratación permite concretar la implementación de las políticas públicas con las que el Estado planifica su actuar (Graglia, 2016).

En este sentido, la construcción de obras públicas, como instrumento de política pública, posee un carácter estratégico orientado a la consecución de resultados (Rezzoagli, 2016); por ende, tiene la posibilidad de incidir directamente en la efectividad de los deberes de protección de la diversidad e integridad del ambiente y de planificación y aprovechamiento de los recursos naturales para garantizar el desarrollo sostenible, señalados en los artículos 79 y 80 de la Constitución Política de 1991. De manera que las obras constituyen un mecanismo ideal para engranar de manera armónica la ya conocida triada del desarrollo sostenible: economía, sociedad y ambiente. 
No obstante, la actividad constructiva tiene grandes implicaciones de orden ambiental dentro de todas sus etapas (planeación, construcción, operación y demolición), debido a que está involucrada en gran medida con la utilización de recursos naturales, como agua, tierra, madera y consumo de energía, y es un alto generador de contaminación por la producción de residuos sólidos, agentes contaminantes y gases de efecto invernadero (Acevedo, Ramírez y Vásquez 2012), lo cual no solamente se relaciona con la actividad de la construcción en sí misma, sino que también involucra los materiales de construcción que, de igual manera, requieren de la extracción y fabricación de materias primas, aunando a su coste energético.

A esto se suma que a partir de la creación del concepto de desarrollo sostenible, aunque se ha evidenciado el aumento en el crecimiento de la economía, así como importantes avances en torno a la erradicación de la pobreza, ha aumentado considerablemente el deterioro ambiental, representado en el desborde de los límites planetarios, principalmente frente al cambio climático y la pérdida de la integridad de la biosfera, de manera que la arista ambiental del desarrollo sostenible, que es justamente la que la diferencia de otros modelos de desarrollo, se ha visto relegada (Rodríguez, 2018), generando por consiguiente un desbalance en las dimensiones que abarcan este principio.

Si bien en el marco de la Ley 80 de 1993 y de la Ley 1150 de 2007 se encuentran disposiciones que están dadas para incluir consideraciones ambientales en todas las fases de la contratación de obras públicas y se ha venido desarrollando el concepto de contratación sostenible (Moreno, 2006), resulta imprescindible aprovechar el carácter estratégico de la contratación estatal para crear mayores impactos en el comportamiento del Estado como consumidor y como actor del mercado, y de esta manera fomentar la producción y 
comercialización de bienes, servicios y, en especial, obras ambientalmente sostenibles que, a su vez, promuevan la oferta de los mismos.

En este orden de ideas y a la luz de los deberes constitucionales establecidos en los artículos 79 y 80 de la Constitución Política de 1991 y del principio de desarrollo sostenible, contemplado en el artículo I de la Ley 99 de 1993, se plantea como pregunta de investigación: ¿el carácter instrumental del contrato de obra pública, para el crecimiento social y económico en Colombia, se ha articulado o no con la aplicación del principio de desarrollo sostenible consagrado en la Constitución Política de 1991 y en la Ley 99 de 1993, desde su dimensión ambiental?

La hipótesis que se formula en este documento de trabajo consiste en que a pesar del carácter instrumental y estratégico del contrato de obra pública para incidir sobre el crecimiento social y económico del país, este no se ha logrado articular con la aplicación del principio de Desarrollo Sostenible consagrado en la Constitución Política de 1991 y en la Ley 99 de 1993 desde su dimensión ambiental, debido a las dificultades para implementar en este contrato las políticas sobre contratación sostenible, el debilitamiento de los instrumentos e instituciones de planificación y gestión ambiental, sumados a la falta de voluntad política para potenciar este tipo de contrato como una herramienta de protección del ambiente.

En este sentido, el objetivo general es determinar si el carácter instrumental del contrato de obra pública para el crecimiento social y económico en Colombia se ha logrado articular o no con la aplicación del principio de desarrollo sostenible, consagrado en la Constitución Política de 1991 y en la Ley 99 de 1993 desde su dimensión ambiental. Para cumplir este objetivo, este escrito se estructura en tres partes que responden a los siguientes objetivos específicos: en primer lugar, identificar cómo se manifiesta el carácter instrumental del contrato de obra pública en el crecimiento social 
y económico del país, a partir de los fines de la contratación estatal establecidos en el artículo 3 de la Ley 80 de 1993.

En segundo lugar, establecer, desde la consagración del principio de desarrollo sostenible en la Constitución Política de 1991 y en la Ley 99 de 1993, los elementos principales que posibilitan la plena aplicación de la dimensión ambiental de este principio en el contrato de obra pública. $\mathrm{Y}$, en tercer lugar, identificar los aspectos que han imposibilitado la articulación del principio de desarrollo sostenible, desde su dimensión ambiental, en el contrato de obra pública.

Para adelantar la presente investigación se utilizó un enfoque hermenéutico crítico, con un método cualitativo, y como estrategia de investigación, se realizó una revisión documental.

\section{El papel del contrato de obra pública como eje estratégico del crecimiento social y económico del país}

Acorde con el artículo 2 de la Constitución Política de 1991, los fines del Estado son variados y para su cumplimiento se requiere del despliegue de todas sus instituciones, para lo cual estas se valen en gran parte de las obras, bienes y servicios que contratan. En particular, los contratos de obra pública son una importante herramienta para el desarrollo de la sociedad, pues en intervienen proyectos de infraestructura, transporte, vivienda, saneamiento etc., fundamentales para el crecimiento de los pueblos (Acevedo, Vásquez y Ramírez, 2012). Es por ello por lo que la presente sección busca identificar cómo se manifiesta el carácter instrumental del contrato de obra pública en el crecimiento social y económico del país, a partir de los fines de la contratación estatal establecidos en el artículo 3 de la Ley 80 de 1993.

Al decir que estos contratos poseen un carácter instrumental para el crecimiento de un país, no se quiere significar que sean un 
mero mecanismo para obtener un producto y así alcanzar de manera abstracta los fines estatales, la prestación de los servicios o la efectividad de los derechos según lo establece el artículo 3 de la Ley 80 de 1993, sino que estos hacen parte de un engranaje complejo de la gestión pública que busca responder a objetivos concretos (Rezzoagli, 2016), resolver problemáticas o garantizar ciertas condiciones de interés del Estado; por ello las obras públicas son una clara expresión de la función administrativa (Aponte, 2014).

Este enfoque se plantea desde lo que se denomina la "nueva gestión pública", que hace referencia a un paradigma de la administración como resultado de la evolución de las diferentes maneras de asumir la administración de lo público aparejadas con los modelos de Estado (Peña, 2016). Así, esta visión de la nueva gestión pública se caracteriza principalmente porque se integran elementos del sector privado a los estándares de la organización y operación de la función administrativa, orientados a la definición de metas, objetivos, maximizar la eficiencia y la obtención de resultados, "significa simplemente la implantación de la visión de los negocios privados dentro del Gobierno" (Peña, 2016, p. 27).

Bajo esta perspectiva, en las sociedades democráticas la función administrativa está llamada a actuar frente a la comunidad, y por ello debe definir cómo incide en las realidades sociales, ya sea a través de los planes de desarrollo gubernamentales o en la satisfacción de intereses puntuales e inmediatos de los administrados (Peña, 2016); es decir, el Estado es el principal responsable de garantizar el bien común, resolver necesidades insatisfechas y generar las condiciones para mejorar la calidad de vida de los asociados, y para alcanzar estos fines debe relacionarse con la ciudadanía y demás actores privados a través de acciones planificadas: políticas públicas (Graglia, 2016).

Conforme con lo anterior, las políticas públicas se estructuran a través de la formulación de planes, y estos se concretan en pro- 
gramas y proyectos, cuyo alcance se identifica por la amplitud de sus objetivos; los planes más amplios se formulan a largo plazo, los programas se establecen a mediano plazo y los proyectos a corto plazo, así, un plan puede incluir programas y estos a su vez proyectos (Graglia, 2016). En Colombia, según el artículo 339 de la Constitución Política de 1991, los propósitos y objetivos nacionales, las prioridades de la acción estatal, las visiones de la política económica, social y ambiental, así como el plan de inversiones públicas deben establecerse mediante el plan nacional de desarrollo, al cual:

le corresponde ejercitar al máximo la planeación, como ciencia y técnica, que permite administrar los principales recursos a ejecutar, bien sean financieros, económicos, humanos, físicos, de información y de conocimiento. Todo este sistema integrado de planeación tiene como objetivo permitir la realización de la función pública con base en la Constitución y la ley, dando un manejo óptimo a los recursos y cumpliéndoles a las comunidades, con la realización de obras prioritarias (Aponte, 2014, p. 188).

Este ejercicio de planificación se manifiesta en la contratación estatal a través del principio de planeación que, si bien no está plasmado expresamente en las normas de contratación pública, es el marco de referencia en el que se articulan las metas y objetivos de los Planes de Desarrollo y se refleja, entre otros aspectos, en la elaboración de los estudios previos, donde las entidades públicas describen la necesidad, conveniencia y oportunidad de la contratación (Aponte, 2014); es decir, en estos documentos se justifica cómo la contratación va a contribuir al cumplimiento de los propósitos y fines establecidos previamente en las instrumentos de planeación estratégica de las entidades.

Puntualmente en las obras públicas, según el artículo 87 de la Ley 1474 de 2011, las entidades deben evaluar, previo a la apertura del proceso, la viabilidad de las obras y su impacto social, económico y ambiental. Esto implica la planeación de la obra con un enfoque 
de proyecto, es decir como un "conjunto de actividades que se desarrollan en forma coherente con el propósito de obtener un resultado final, como respuesta a una necesidad u oportunidad de negocio, en un tiempo determinado y mediante la utilización de recursos” (Díaz, Murcia, Onate, Rodríguez, Rodríguez, Rojas y Santana, 2019 , p. 29), propio de las ciencias administrativas y de ingeniería, pero que igualmente debe alinearse con los planes de desarrollo y los propósitos estratégicos de una organización (Díaz et al., 2019).

Ahora bien, esta labor de planificación funciona en la medida en que las políticas públicas se puedan implementar de forma que efectivamente se cumplan las metas y objetivos propuestos; y la manera como se ejecuta la planificación, es convirtiendola en actividades que se manifiestan en el desarrollo de obras y la prestación de servicios. Por ello se dice que los planes y las actividades son elementos necesarios para la existencia de las políticas públicas (Graglia, 2016). Así, la contratación pública deja de verse como un conjunto de procedimientos y de reglas para convertirse en una herramienta estratégica de política pública orientada a resultados (Rezzoagli, 2016).

No obstante, las actividades, a través de las cuales se evidencia el cumplimiento de las políticas, no implican necesariamente que el Estado sea el único responsable en su realización. Como se indicó líneas arriba, la acción planificada del Estado involucra la relación con la sociedad, pues es a esta a quien va dirigida esa acción, y en ese sentido, esta misma acción, como puede ser el caso de la construcción de obras, también puede encargarse a particulares, sin que el Estado se desligue de su obligación en el resultado de las metas y objetivos definidos en sus planes (Graglia, 2016).

Esta idea se encuentra plasmada en el inciso segundo del artículo 3 de la Ley 80 de 1993, al señalar que, cuando los particulares contratan con el Estado, contribuyen con el logro de sus fines y cumplen una función social. De esta forma, se puede establecer 
que los contratos de obras públicas son a la vez un mecanismo de interlocución directa entre este y los particulares como actores en la construcción del bienestar general de la sociedad. Esta relación de doble vía también refleja el espíritu democrático de la función administrativa que caracteriza el Estado social y de derecho (Araújo, 2011).

Esta interacción entre el Estado y los particulares como destinatarios y partícipes en la contratación estatal no solamente se manifiesta como elemento de la función administrativa, como se ha expuesto, sino que también permite incidir en la dinámica económica del país, al promover la producción y comercialización de bienes y servicios, por lo tanto se constituye como una herramienta de desarrollo, pues también involucra a los actores vulnerables de la comunidad dentro del escenario económico, favorece la generación de empleo y el desarrollo productivo (Sela, 2014). Así, dado que en la contratación pública tiene una importante participación dentro del gasto público, resulta determinante para el crecimiento del país y de la región:

[a] través del gasto público, los gobiernos implementan las políticas públicas y los planes de desarrollo; afectan la demanda agregada, el crecimiento económico y el empleo; condicionan la estabilidad macroeconómica y contrarrestan fallas del mercado. En América Latina y el Caribe, las compras públicas de bienes y servicios representaron, para el período 1990-2009, una cifra entre el 10 y el 15\%7 del gasto público regional (Sela, 2014, p. 16).

De acuerdo con lo anterior, se puede decir que la contratación de obras públicas son un arquetipo de la generación de impacto social (Aponte, 2014), pues en ellas se inscriben sectores como, por ejemplo, el de la infraestructura que, dentro de la contratación pública, es una de las actividades más relevantes al momento de llevar a cabo políticas públicas necesarias para alcanzar el desarrollo (Sela, 
2014), y por ello es que las obras públicas también se relacionan inevitablemente con la industria de la construcción como sector de la economía y ciertamente tienen una incidencia directa en su comportamiento ${ }^{2}$.

En consideración a todo lo expuesto, se puede indicar a modo de conclusión que el carácter instrumental del contrato de obra pública, a la luz de los fines de la contratación estatal del artículo 3 de la Ley 80 de 1993, se manifiesta en que más allá de considerarse como un simple medio para la adquisición de un producto final, a partir de la óptica de la nueva gestión pública, es un elemento estratégico de las políticas públicas porque, al ser un contrato estatal, en él se concretan y se cumplen las metas y objetivos establecidos en la planificación de dichas políticas que se orientan a resolver problemáticas específicas, satisfacer intereses comunes y garantizar condiciones de bienestar.

Por ello, la contratación de obras públicas es una manifestación de la función administrativa contemplada en el artículo 209 de la Constitución Política de 1991, con un carácter participativo propio del Estado social de derecho, que involucra a los particulares y a los sectores vulnerables no solo como objeto de atención estatal, sino además como colaboradores en el cumplimiento de los propósitos que se buscan atender con la ejecución de la contratación, tal como

2 Acorde con Procolombia (2016), este sector en general ha contribuido al crecimiento del país, pues aumentó cerca del $8 \%$ entre 2009 y 2013 y logró ubicarse en el tercer lugar en Latinoamérica, después de Brasil y México, aportando USD 35000 millones en 2014 al PIB de la región. A su vez, los proyectos de vivienda promovidos por el gobierno nacional y de infraestructura a través de programas de concesiones de carreteras de cuarta generación (4G) han contribuido al impulso del sector en general y de hecho son incentivos que ha utilizado el Estado para atraer la inversión extranjera hacia este sector. Aún cuando el sector de la construcción ha venido presentando contracciones económicas desde el año pasado (Banco de la República, 2019), el impulso de la inversión estatal en proyectos, como por ejemplo de infraestructura y vivienda (Santa María, 2020), demuestran el factor determinante de estos contratos en la recuperación económica en el sector de la construcción, en especial, en la coyuntura actual causada por la pandemia del COVID-19. 
lo establece el artículo 3 de la Ley 80 de 1993; en esa interacción, la contratación también participa en procesos de desarrollo y es un dinamizador del crecimiento de los sectores económicos en los que se involucra.

De esta manera, si bien la contratación de obras públicas resulta ser un actor fundamental en los procesos de desarrollo del país, no es menos cierto que, a la vez, esta actividad está relacionada con la generación de impactos negativos al ambiente, el alto nivel de recursos naturales que consume la actividad constructiva, la contaminación que produce, entre otros aspectos. Es por ello por lo que, conforme con el principio del desarrollo sostenible consagrado en la Constitución Política de 1991 y en la Ley 99 de 1993, resulta indispensable establecer en la siguiente sección los principales elementos que posibilitan la plena aplicación este principio desde su dimensión ambiental en el contrato de obra pública.

\section{Los principales elementos de gestión ambiental en el contrato de obra pública}

Como se vió en la sección anterior, el contrato estatal de obra se erige como un instrumento estratégico de política pública para el logro del desarrollo del país y de los fines estatales establecidos en la Constitución Política de 1991. Ahora, en la presente sección se pretende, desde la consagración del desarrollo sostenible como principio general de derecho y eje temático de la constitución ecológica de Colombia (Amaya, 2016), principio que también fue acogido en la Ley 99 de 1993, establecer los elementos principales que posibilitan la plena aplicación de la dimensión ambiental de este principio en el contrato de obra pública.

Para empezar, es preciso señalar antes que el desarrollo sostenible, aunque no está propiamente definido en el artículo 80 de la Constitución Política de 1991, sí se encuentra en el artículo 3 de la 
Ley 99 de $1993^{3}$, además en el artículo 1 se acogió este principio desde el contenido previsto en la Declaración de Río de 1992 sobre medio ambiente y desarrollo. Este contenido ha tenido una suerte de evolución que ha permitido fortalecer su fundamento (Rodríguez, 2018), por lo que es importante hacer una breve aproximación de la arista ambiental del desarrollo sostenible con las últimas precisiones sobre su conceptualización, que contribuirá a entender la aplicación de dicha arista en el contrato de obra pública.

A partir de la concepción del desarrollo sostenible en la Declaración de Río, se consolidaron algunos elementos que se venían estructurando con anterioridad ${ }^{4}$, principalmente en el principio $4^{5}$ de la declaración, se lograron integrar las preocupaciones frente a garantizar el desarrollo económico, la lucha contra la pobreza y la protección ambiental (Rodríguez, 2018), estableciendo las dimensiones económica, social y ambiental del desarrollo sostenible frente a las cuales:

Al tratarse de un principio jurídico que pretende ser un criterio objetivo de conducta se espera que solo refleje una realidad. Así pues, no se considera válida y viable una valoración del desarrollo sostenible desde sus dimensiones, sino por el contrario que las tome todas en cuenta de manera neutra (Gómez, Monroy y Rodríguez, 2013, p. 32).

Asimismo, en esta declaración se inscribió al hombre como centro de las preocupaciones asociadas con el desarrollo sostenible

3 Artículo 3 Ley 99 de 1993: Se entiende por desarrollo sostenible el que conduzca al crecimiento económico, a la elevación de la calidad de la vida y al bienestar social, sin agotar la base de recursos naturales renovables en que se sustenta, ni deteriorar el medio ambiente 0 el derecho de las generaciones futuras a utilizarlo para la satisfacción de sus propias necesidades.

4 La Declaración de Estocolmo de 1972 sobre medio ambiente humano, el informe del Club de Roma del mismo año denominado "Los límites del crecimiento", así como el informe de 1987 de la Comisión Brundtland son los antecedentes más reconocidos que contribuyeron a la conformación del contenido del desarrollo sostenible (Rodríguez, 2018).

5 Principio 4: A fin de alcanzar el desarrollo sostenible, la protección del medio ambiente deberá constituir parte integrante del proceso de desarrollo y no podrá considerarse en forma aislada (Naciones Unidas, s.f.a). 
(principio 1)6, adoptando una connotación antropocéntrica del desarrollo (Gómez, Monroy y Rodríguez, 2013), el concepto intergeneracional en la satisfacción de las necesidades (principio 3)7 y la erradicación de la pobreza como requisito para alcanzar este modelo de desarrollo (principio 5)8, por nombrar los elementos más destacados. Si bien se han presentado críticas y debates frente a esta concepción del desarrollo sostenible (Rodríguez, 2018, Gómez, 2009), esta visión ha sido generalmente aceptada por los gobiernos, sectores empresariales y parte de la academia (Rodríguez, 2018).

Adicionalmente, se han incorporado otros dos elementos fundamentales para entender la arista ambiental del desarrollo sostenible. El primero hace referencia a los servicios ecosistémicos que dan soporte al funcionamiento de la tierra como: procesos biofísicos de los suelos, ciclos de nutrientes, servicios de aprovisionamiento y regulación y servicios, como por ejemplo recreativos, culturales o religiosos, que se relacionan con la calidad de vida de los seres humanos. En este sentido, se presenta una interrelación entre los impactos que estos servicios generan sobre la actividad humana y viceversa, y esta interrelación tiene la capacidad de generar beneficios o amenazas sobre ambos (Rodríguez, 2018). El segundo elemento se refiere a los nueve límites planetarios, a saber:

cambio climático, cambio en la integridad de la biosfera, eliminación del ozono estratosférico acidificación de los océanos, ciclos biogeoquímicos (ciclos de nitrógeno y de fósforo), cambio de los sistemas de

$6 \quad$ Principio 1: Los seres humanos constituyen el centro de las preocupaciones relacionadas con el desarrollo sostenible. Tienen derecho a una vida saludable y productiva en armonía con la naturaleza (Naciones Unidas, s.f.a).

7 Principio 3: El derecho al desarrollo debe ejercerse en forma tal que responda equitativamente a las necesidades de desarrollo y ambientales de las generaciones presentes y futuras (Naciones Unidas, s.f.a).

8 Principio 5: Todos los Estados y todas las personas deberán cooperar en la tarea esencial de erradicar la pobreza como requisito indispensable del desarrollo sostenible, a fin de reducir las disparidades en los niveles de vida y responder mejor a las necesidades de la mayoría de los pueblos del mundo (Naciones Unidas, s.f.a). 
suelo, uso del agua dulce, la carga de aerosoles en la atmósfera y la carga de químicos. (Rodríguez, 2018, p. 14-15).

Estos límites se encuentran íntricamente relacionados entre sí y a su vez se conectan con los servicios ecosistémicos, lo anterior se explica porque al transgredir los límites a una escala planetaria se afectan a su vez los servicios ecosistémicos, lo cual deriva en procesos con graves consecuencias para el sistema terrestre y quienes habitan en él, como los que se están empezando a evidenciar, siendo el caso de la sexta extinción de las especies (por primera vez a causa de la actividad humana) y los fenómenos climáticos anormales: descongelamiento de los glaciales y fuertes periodos invernales o de sequías (Rodríguez, 2018).

Para finalizar esta aproximación al contenido último del desarrollo sostenible, es necesario mencionar los objetivos de desarrollo sostenible (ODS). Se trata de un enfoque renovado del desarrollo basado en objetivos que resultó de la evolución de los objetivos de desarrollo del milenio ODM y de la Agenda 21, cuya orientación estaba sectorizada respectivamente a los países pobres y a los países en desarrollo, (Rodríguez, 2018). Así los ODS proponen una nueva agenda global que incluye también a los países ricos y otros actores de la comunidad global, donde se destaca esta vez a la buena gobernanza como un componente que interactua con la triada economía, sociedad y ambiente (Sach, 2014).

Así las cosas, los estados miembros de la Organización de las Naciones Unidas establecieron $17 \mathrm{ODS}^{9}$ a 2030 con los que

9 Los objetivos son los siguientes: 1) Fin de la pobreza; 2) Hambre cero; 3) Salud y bienestar; 4) Educación de calidad; 5) Igualdad de género, 6) Agua limpia y saneamiento; 7) Energía asequible y no contaminante; 8) Trabajo decente y crecimiento económico; 9) Industria, innovación e infraestructura; 10) Reducción de las desigualdades; 11) Ciudades y comunidades sostenibles; 12) Producción y consumo responsables; 13) Acción por el clima, 14) Vida submarina; 15) Vida de ecosistemas terrestres; 16) Paz, justicia e instituciones sólidas y 17) Alianzas para lograr los objetivos (Naciones Unidas, s.f.b). 
se logró dar una prioridad adecuada a la dimensión ambiental (Rodríguez, 2018) y que de manera puntual buscan alcanzar los propósitos del desarrollo sostenible. De esta forma, este nuevo enfoque facilita la movilización social hacia acciones unificadas, el cumplimiento de las metas trazadas por la presión grupal, la participación y asesoría de comunidades epistémicas o redes de conocimientos, saberes, prácticas y de actores de interés $(\mathrm{ONG}$, sector académico, líderes políticos, comunitarios y religiosos, entre otros) para el logro de los objetivos (Sach, 2014).

Bajo este contexto y retomando la consagración del principio de desarrollo sostenible en la Constitución Política de 1991, este resulta ser "un modelo económico compatible con la protección y preservación de los recursos naturales" (Amaya, 2006, p. 19), por ello, el artículo 80 estableció la obligación a cargo del Estado de planificar el manejo y aprovechamiento de los recursos naturales y en este sentido, la contratación estatal no es ajena a este deber como quiera que el aprovisionamiento de bienes, obras y servicios para la satisfacción de las necesidades al momento de contratar, supone el uso y disposición de estos recursos, y por consiguiente está asociada con los impactos ambientales, que tales aprovisionamientos genera.

Uno de los elementos más importantes, que responde a este deber de garantizar el desarrollo sostenible en el marco del contrato estatal de obra, es la evaluación de impacto ambiental como una herramienta de planificación y gestión ambiental (Gómez, Monroy y Rodríguez, 2013). De esta manera, el numeral 11 del artículo 1 de la Ley 99 de 1993 estableció como principio de la política ambiental los estudios de impacto ambiental, para la toma de decisiones en la construcción de obras cuando afecten significativamente el ambiente 
natural o artificial. Este estudio se concreta principalmente en las licencias ambientales creadas por el artículo 50 de la referida Ley ${ }^{10}$.

Para Gómez, Monroy y Rodríguez (2013), la licencia ambiental es la materialización de la evaluación ambiental, que se orienta hacia el desarrollo sostenible a un largo, mediano y corto plazo, con el propósito de reducir los efectos que un proyecto puede generar desde el punto de vista biofísico, biótico, abiótico, económico, social y cultural; igualmente, afirman que la licencia es un medio para garantizar la protección de los derechos colectivos ambientales en línea con las obligaciones de orden constitucional y con los demás principios en materia ambienta ${ }^{11}$. Asimismo, son consideradas como un proceso que permite que las actividades licenciadas se sujeten a los límites ecológicos, siendo una herramienta fundamental para impulsar el desarrollo sostenible.

Esta herramienta de gestión ambiental también se encuentra asociada con el principio de planeación de la contratación estatal,

10 Artículo 50 Ley 99 de 1993: Se entiende por Licencia Ambiental la autorización que otorga la autoridad ambiental competente para la ejecución de una obra o actividad, sujeta al cumplimiento por el beneficiario de la licencia de los requisitos que la misma establezca en relación con la prevención, mitigación, corrección, compensación y manejo de los efectos ambientales de la obra 0 actividad autorizada. A partir de esta Ley la licencia ha tenido un extenso desarrollo normativo, así como cambios en su contenido. Actualmente es regulada por el Decreto Único Reglamentario del Sector Ambiente y Desarrollo Sostenible 1076 de 2015, modificado parcialmente en materia de licencias y permisos ambientales para Proyectos de Interés Nacional y Estratégicos (PINE) por el Decreto 2220 de 2015.

11 Estos principios están establecidos en la Constitución Política de 1991 y en la Ley 99 de 1993 y se encuentran relacionados con la licencia ambiental. Principalmente (además del desarrollo sostenible) son: medio ambiente sano, precaución, prevención, el que contamina paga, armonía regional, gradación normativa, rigor subsidiario, participación y acceso a la información y evaluación de impacto ambiental. Adicionalmente, en la actualidad tienen competencia para conocer del trámite de las licencias la Autoridad Nacional de Licencias Ambientales (ANLA), las Corporaciones Autónomas Regionales y de Desarrollo Sostenible, los Grandes Centros Urbanos y los Distritos de Barranquilla, Santa Martha y Cartagena (Gómez, Monroy y Rodríguez, 2013). 
pues a la luz del artículo 87 de la Ley 1474 de 2011 y del artículo 2.2.1.1.2.1.1. del Decreto 1082 de 2015, las entidades deben considerar en sus documentos previos los impactos ambientales que se pueden generar por el contrato de obra, así como también deben identificar los permisos y licencias requeridos para la ejecución del contrato, además, "[1]a planeación debe observarse en cada una de las etapas de desarrollo de la obra, del cumplimiento del contrato y la entrega del bien, contemplando además la atención de la carga ambiental y el cumplimiento de las garantías” (Aponte, 2014).

La licencia ambiental constituye entonces un elemento primordial para aplicar el principio de desarrollo sostenible, dado que la evaluación de los impactos ambientales de la obra o proyecto, permite realizar una valoración de los servicios ambientales involucrados y de los límites ecológicos que podrían verse alterados, para implementar las medidas de prevención, protección, manejo y compensación de los ecosistemas y recursos naturales afectados (Gómez, Monroy y Rodríguez, 2013), lo cual es un ejercicio de planificación ambiental en línea con lo ordenado por el artículo 80 de la Constitución Política de 1991, y que en el contrato de obra se logra concatenar con la aplicación del principio de planeación que, en su carácter dinámico, irradia todas las etapas del contrato para asegurar el cumplimiento del objeto contractual y de los objetivos que se buscan con la contratación (Aponte, 2014).

Por otra parte, en concordancia con los procesos de planificación ambiental que se formulan en armonía con lo previsto en los artículos 80 y 339 de la Constitución Política de 1991 para asegurar el desarrollo sostenible (Rodríguez, 2012), se pretende destacar en este punto un elemento que busca conjugar la gestión ambiental con la contratación estatal, para estimular el cambio en los patrones de producción y consumo en el mercado de obras, bienes y servicios que se demandan y se ofertan en las entidades públicas, y que se inscribe dentro del ODS n. 12 de producción 
y consumo responsables ${ }^{12}$, se trata de la contratación sostenible o compra pública sostenible.

La contratación sostenible se refiere a la introducción de criterios ambientales en los contratos a partir de herramientas jurídicamente vinculantes y aspectos técnicos ambientales acordes con la tipología contractual y en armonía con los derechos y obligaciones que se derivan de las mismas (Moreno, 2006). En el marco de la contratación pública, esta herramienta nació como producto de una serie de acciones de planificación que inició en el contexto nacional con la adopción de la Política Nacional de Producción y Consumo Sostenible y se ha desarrollado a través del Plan de Acción Nacional de Compras Públicas Sostenibles y el Documento CONPES 3919 de 2018 sobre edificaciones sostenibles ${ }^{13}$.

Como resultado de estas acciones, Colombia Compra Eficiente expidió la Guía de compras públicas sostenibles con el ambiente, que define estas compras como un proceso de compra, en donde se satisfacen las necesidades y, a la vez, se propende una reducción de los recursos a

12 Acorde con el Ministerio de Ambiente y Desarrollo Sostenible (2017), la meta 12.7 de este ODS señala que los gobiernos deben garantizar prácticas de contratación pública sostenible acordes con las prioridades y políticas nacionales.

13 Desde el Ministerio de Ambiente y Desarrollo Sostenible también se han elaborado otros documentos estratégicos como la guía conceptual y metodológica de las compras públicas sostenibles, el manual de compras públicas sostenibles para entidades públicas y privadas y el aplicativo de fichas técnicas con criterios de sostenibilidad y análisis de costo beneficio, este último desarrollado con el Centro Nacional de Producción más Limpia (Ministerio de Ambiente y Desarrollo Sostenible, s.f.). Igualmente se creó el Sello Ambiental Colombiano (SAC), mediante la Resolución 1555 de 2005, como un sistema de reconocimiento voluntario con el objetivo de brindar una información exacta y no engañosa a los consumidores, sobre las propiedades ambientales de los productos que se someten al sello ambiental. (Ministerio de Ambiente y Desarrollo Sostenible, s.f.). Con el establecimiento del SAC se reconoce la preocupación por parte del sector productivo de armonizar sus procesos de producción con técnicas o procedimientos más amigables con el ambiente, sin dejar de lado el valor económico del producto, poder en el mercado o disminución en la producción (Briceño, 2008). Actualmente, se cuenta con 23 categorías (normas técnicas) para optar por el SAC, dentro de las cuales las siguientes se relacionan con la construcción de obras: NTC-6093-SAC prefabricados concreto, NTC-6034-SAC elementos de acero planos conformados en frío para uso en construcción, NTC-6033-SAC ladrillos y bloques de arcilla, NTC-6024-SAC baldosas cerámicas y NTC-6018-SAC pinturas y materiales de recubrimiento. 
través de la consideración de los impactos ambientales de la contratación y el análisis de todo el ciclo de vida del bien o servicio a adquirir (Colombia compra eficiente, s.f.). En dicha guía, a partir de un contexto sobre los beneficios de estas compras y del marco legal aplicable ${ }^{14}$,

14 Es conveniente precisar que desde la Ley 80 de 1993 hay un marco jurídico que siempre ha permitido el establecimiento de componentes ambientales, pero es gracias a este desarrollo de política que puede ver como un ejercicio consciente y con un propósito estratégico. Frente a esta Ley, puede decirse que en materia ambiental se contemplaron dos disposiciones explícitas en este campo: la primera es el parágrafo segundo del artículo 32 (derogado posteriormente por el artículo 39 de la Ley 1508 de 2012, que estableció el régimen jurídico de las Asociaciones público privadas), al referirse a los estudios de impacto ambiental que debían incluirse en las ofertas para los contratos de concesión para la construcción de obras públicas, y la segunda, está contenida en el inciso segundo del artículo 65 frente a las autoridades que tienen a su cargo el control fiscal, las cuales deben realizar la vigilancia entre otros aspectos, sobre la valoración de los costos ambientales de los contratos terminados o liquidados. Esta última disposición es una clara manifestación del principio de gestión fiscal contenido en el artículo 267 de la Carta Política, que hace parte de las disposiciones que conforman la llamada Constitución verde. De otro lado, se puede decir que aunque no de una forma expresa, la Ley 80 de 1993 se encuentra también relacionada con el amparo de los derechos o deberes relativos al ambiente, cuando determina que el propósito mismo de los contratos se orienta a la consecución de los fines estatales (artículo 3), o cuando dispone una serie de deberes y responsabilidades que eventualmente podrían ser de tipo ambiental: adelantar acciones por indemnización de daños que sufra la Entidad con ocasión del contrato (artículo 4, numeral 6); deber de los servidores públicos de proteger los derechos de terceros que puedan verse afectados con la ejecución del contrato y su responsabilidad por sus actuaciones y omisiones antijurídicas, con lo cual están obligados a indemnizar los daños que se causen por razón de ellas (artículo 26, numeral 1 y 2). Otra de las previsiones que encuentra relación con el tema ambiental, tiene que ver con la verificación de requisitos habilitantes, en el proceso de selección de contratistas, Así, la Ley 1150 de 1997 estableció una diferenciación entre los requisitos mínimos habilitantes y los factores de selección, siendo los primeros aquellos que validan la experiencia y elementos de capacidad financiera, técnica, organizacional y jurídica del oferente, que no otorgan puntaje sino que lo habilitan para permitir la evaluación objetiva de su oferta; y los segundos, los que a partir de criterios económicos y técnicos, aplicados según las fórmulas de ponderación señaladas en los pliegos de condiciones o sus equivalentes, permiten seleccionar la oferta más favorable para la Entidad, de manera que si bien no se expresa directamente, el Estatuto permite la inclusión de criterios de sostenibilidad ambiental en las especificaciones técnicas de los bienes y servicios a contratar a través de los procesos de selección, de manera que se armoniza el principio de selección objetiva contenido en el artículo 5 de la Ley 1150 de 2007, con la valoración de los costos ambientales a que se refiere el principio de gestión fiscal consagrado en el artículo 267 de la Constitución (Ministerio de Ambiente, Vivienda y Desarrollo Territorial, 2010). Otro punto importante, es que, a partir de la Ley 1150 de 2007, se introdujo el deber de incluir la estimación, tipificación y asignación de los riesgos previsibles en Ios pliegos definitivos o sus equivalentes. De acuerdo con el documento CONPES 3714 de 2011, en el que se establecieron varios lineamientos básicos para el manejo de los riesgos, en la fase de tipificación de los riesgos se agrupó de manera enunciativa una propuesta de clasificación de estos, y en esta clasificación se incluyeron los riesgos ambientales. igualmente, en la fase de asignación de los riesgos, con base en la clasificación propuesta, el documento también planteó la distribución de los riesgos ambientales en cinco diferentes escenarios. 
dota a las entidades de herramientas para implementar consideraciones ambientales en sus adquisiciones. No obstante, esta tarea implica un ejercicio de proporcionalidad y pertinencia que también garantice los principios de selección objetiva y pluralidad de oferentes (Moreno, 2006).

De esta forma para Moreno (2006) es necesaria la priorización de los contratos en los que sea verdaderamente factible la introducción de criterios ambientales, el análisis de los impactos ambientales de las condiciones técnicas de la contraprestación, la estructuración de los criterios ambientales para la selección de contratistas que incida en la propuesta más favorable en el marco de la selección objetiva. En este caso, propone la incorporación en el registro único de proponentes (RUP) de información ambiental del proveedor que permita su comparación con otros oferentes, así como de datos sobre procesos sancionatorios en trámite o sanciones ya impuestas por autoridades ambientales.

De acuerdo con lo expuesto a lo largo de esta sección, puede decirse que el marco del desarrollo sostenible establecido en la Constitución Política de 1991 y la Ley 99 de 1993, junto con la conceptualización que ha enriquecido su contenido, existen dos elementos principales que posibilitan la plena aplicación de la dimensión ambiental de este principio en el contrato de obra pública. El primero de ellos es la licencia ambiental como herramienta fundamental para la gestión y toma de decisiones. Además, al ser un instrumento de planificación ambiental se encuentra directamente asociado con los proceso de planeación en los procesos de contratación, a la luz del principio de planeación.

El segundo es la contratación pública sostenible; gracias a los avances de los instrumentos de política pública se dota a las entidades estatales de bases y metodologías para incluir criterios de sostenibilidad ambiental en sus contrataciones, en cumplimiento del ODS 
12, lo cual tiene un especial relevancia en el contrato de obra, dado que la implementación de características técnicas ambientalmente sostenibles en los insumos o procesos de producción o construcción de obras resulta fundamental para mejorar la eficiencia en el uso o explotación de los recursos naturales al considerar los ciclos de vida de tales insumos o procesos que abarcan incluso hasta la disposición final de los mismos.

A pesar de lo anterior, el panorama actual vislumbra una crisis ambiental profundizada principalmente por la pérdida de diversidad y el cambio climático, lo que hace pensar en un atraso de la dimensión ambiental del desarrollo sostenible, que impide el avance equilibrado de la triada economía, sociedad y ambiente. Por lo cual, teniendo el contrato estatal de obra un carácter instrumental para alcanzar el desarrollo y visto que existen en él valiosos elementos que posibilitan esta dimensión ambiental, es necesario ahora identificar aquellos aspectos que han imposibilitado la articulación del principio de desarrollo sostenible, desde su dimensión ambiental, en el contrato de obra pública.

\section{La desarticulación de la dimensión ambiental del principio de desarrollo sostenible, en el contrato de obra pública}

La Constitución política de Colombia de 1991, la Ley 99 de 1993, la Ley 80 de 1993 y la Ley 1150 de 2007 constituyen un marco jurídico que permitió el establecimiento de la licencia ambiental y la contratación sostenible como dos importantes elementos para aplicar la dimensión ambiental del desarrollo sostenible en el contrato de obra pública, pues suponen la valoración de los impactos ambientales asociados a la contratación y aportan herramientas para administrar tales impactos de manera que se garantice una correcta planificación y protección de los recursos naturales; sin embargo, el aumento en el deterioro de dichos recursos, hace pensar que existen 
fallas en la aplicación de estos elementos, por lo cual es importante identificar los aspectos que han imposibilitado la articulación del principio de desarrollo sostenible, desde su dimensión ambiental, en el contrato de obra pública.

Debido a que la construcción de obras está asociada al uso de recursos naturales, generación de residuos, contaminación y transformaciones al entorno, es causante de importantes impactos que pueden afectar el ambiente y sus recursos naturales (Acevedo, Ramírez y Vásquez 2012), es por ello por lo que, como se vio en la sección anterior, la Ley 99 previó las licencias ambientales como instrumento de evaluación para medir aquellos impactos que pueden producir daños al ambiente. Sin embargo, la regulación de estas licencias ha sido objeto de un proceso de flexibilización, como consecuencia de una extensa producción normativa que ha llevado al menoscabo de su rigurosidad (Gómez, Monroy y Rodríguez, 2013; Sleman y Velásquez 2016) $)^{15}$.

Dentro de este proceso de flexibilización, se observa una tendencia hacia la disminución de las actividades sujetas a licencia, como, por ejemplo, las obras públicas de la red fluvial excluidas por el Decreto 1180 de 2003, las obras en municipios que no cuentan con un plan de ordenamiento territorial aprobado, retiradas por el Decreto 1220 de 2005 (Gómez, Monroy y Rodríguez, 2013), o en el caso de proyectos de infraestructura de transporte, la Ley 1682 de 2013 excluyó del licenciamiento las obras de mantenimiento, rehabilitación y mejoramiento, lo cual generó que:

15 De acuerdo con estos autores las principales normas a partir de la Ley 99 de 1993, que se han expedido para reglamentar el licenciamiento ambiental en Colombia, son las siguientes: Decreto 1753 de 1994, Decreto 1728 de 2002, Decreto 1180 de 2003, Decreto 1220 de 2005, 2820 de 2010, Decreto 2041 de 2014 y Decreto 1076 de 2015. Adicionalmente, la Ley 1682 de 2013 contiene algunas previsiones sobre el licenciamiento en proyectos de infraestructura de transporte, al igual que el actual Plan Nacional de Desarrollo, Ley 1955 de 2019, contiene varias disposiciones en esta materia. 
gran cantidad de proyectos de infraestructura del transporte priorizados por el Plan de Desarrollo son presentados hoy, a pesar de su impacto e importancia, como meros mejoramientos, rehabilitaciones o mantenimientos, cuando en esencia, implican la realización de nuevos proyectos que deberían estar sujetos a evaluación ambiental (Sleman y Velásquez 2016, p. 507).

Lo que resulta preocupante, no solo por la disminución del listado de actividades que deben contar con licencia ambiental, es que este proceso de desmonte no cuenta con un soporte técnico que con rigor justifique los efectos ambientales que puede generar el retiro de la obligatoriedad de la licencia (Sleman y Velásquez, 2016) y, de igual manera, tampoco se evidencia una tendencia a incluir nuevas actividades que a largo plazo pueden traer efectos significativos al ambiente y que deberían ser contemplados (Gómez, Monroy y Rodríguez, 2013); con lo cual, en materia de contratación, la etapa de planeación pierde una herramienta fundamental para valorar los impactos ambientales que pueden comprometer el cumplimiento del desarrollo sostenible, particularmente, en sus concepción intergeneracional, de servicios ecosistémicos y límites ambientales.

Ahora bien, más allá de la discusión de la flexibilización de actividades sujetas a licencias, también se presentan otras debilidades que tiene que ver con la baja calidad de la evaluación ambiental debido a que paulatinamente se han reducido los términos para expedir la licencia; la evaluación de impacto ambiental carece de un análisis interdisciplinario; hay una escasa participación de la sociedad civil; las autoridades ambientales han dado más prioridad a las exigencias de sus procesos de gestión de calidad que al propósito de la licencia; asimismo, en la etapa de seguimiento a los proyectos, se denota un insuficiente control a los mismos, por ejemplo, no se adelantan visitas intempestivas para verificar el cumplimiento real de la licencia (Gómez, Monroy y Rodríguez, 2013). 
Aunado a lo anterior, los procesos de participación ciudadana y de consulta previa son débiles, pues no se estimula el compromiso de la sociedad civil en hacer parte e informarse sobre los trámites de licencia que se adelantan dentro de su comunidad y, en los casos donde existe interés, los aportes de quienes participan no tienen mayor incidencia ni son determinantes en las decisiones del proceso de licenciamiento ambiental (Gómez, Monroy y Rodríguez, 2013). Así, esta falencia puede repercutir negativamente en un contrato de obra, dado que un inadecuado o nulo proceso de participación puede generar que la obra no se ejecute, pues:

de no efectuarse este proceso de cambio en materia de democracia participativa, no sólo aumentarán los costos económicos sino también los sociales, políticos e institucionales, poniéndose en riesgo los mismos proyectos, demorando su desarrollo, afectando las inversiones en las diferentes regiones del país y escalando en los conflictos ambientales (Gómez, Monroy y Rodríguez, 2013, p. 195).

Es posible que estas falencias en las licencias también sean producto del debilitamiento de la institucionalidad, en tanto que, desde el establecimiento del Sistema Nacional Ambiental en 1993, las autoridades ambientales no han logrado consolidarse con éxito debido a la inestabilidad en su gestión, agenda estratégica, posicionamiento político, la alta rotación de sus directivos, pérdida de personal calificado y experimentado, la reducción de sus recursos presupuestales, la falta de una gestión eficaz para ejercer control en el territorio nacional (Guhl, 2015; Mance, 2008; Contraloría General de la República, 2017), así como la carencia de articulación interinstiucional para coordinar los usos del suelo establecidos en los planes de ordenamiento territorial, los planes de gestión ambietnal regional y los planes de ordenación y manejo de cuencas hidrográficas (Gómez, Monroy y Rodríguez, 2013).

De acuerdo con Sleman y Velásquez (2016) y Guhl (2015), las dificultades que se presentan en el licenciamiento también se explican 
por una visión cortoplacista del desarrollo por parte de los gobiernos de turno, en la que los recursos naturales (cuyo uso se somete a licenciamiento) son concebidos como bienes económicos (Munévar y Valencia, 2020), de manera que los instrumentos de planificación y protección ambiental son vistos como un obstáculo a la modernización y a los beneficios económicos. Esta percepción puede entrar en conflicto con quienes perciben dichos recursos como derecho colectivo objeto de conservación, es decir, se contraponen diferentes valoraciones del ambiente y de los derechos que surgen de él, en donde:

[t]odo acto de resistencia en contra de estas formas jurídicas de administración genera luchas desiguales y reducen a las partes en conflicto a un antagonismo de polarización, por medio de la cual se entiende que los actores que no están a favor del Estado y sus normas y políticas están en contra de él. Esa concepción política de los conflictos socio-ambientales lucha contra la barrera de la legalidad, pero a su vez devela el carácter ilegitimado del Estado como actor y generador (Munévar y Valencia, 2020, p. 60).

En síntesis, hoy por hoy las licencias ambientales presentan graves deficiencias debido a la falta de seguridad jurídica producto de su inestable regulación y a que se ha desnaturalizado el propósito para la que fueron establecidas (Munévar y Valencia, 2020). Su desmonte progresivo ha desorientado su finalidad como verdaderas herramientas de planificación ambiental, a meros trámites administrativos (Gómez, Monroy y Rodríguez, 2013), por lo que en el marco de las obras públicas, esta situación dificulta que se haga efectivo su carácter estratégico para la conservación de los recursos naturales como elemento integrante de la arista ambiental del desarrollo sostenible y se pierda el valor democrático de la participación de los administrados como actores y destinatarios de las políticas públicas que se buscan materializar con la ejecución de las obras públicas.

Tal vez el mayor avance que se ha realizado últimamente para facilitar el desarrollo sostenible y fortalecer la protección ambiental a 
través de la contratación pública, tal como se trató en la sección anterior, han sido las compras públicas sostenibles como acción consciente de su rol estratégico en el desarrollo del país, pues ha dotado de importantes políticas, metodologías y herramientas para inducir a las entidades públicas a que incorporen criterios ambientales en sus contrataciones, todo sobre la base de un marco constitucional y legal que es completamente abierto para la introducción de estos criterios en la configuración del proceso contractual y del contrato mismo (Moreno, 2006).

Sin embargo, este avance es aún incipiente, en primer lugar, porque la definición de criterios ambientales es una operación delicada, pues están en juego principios de la contratación, como la selección objetiva y la pluralidad de oferentes, de modo que no es fácil para las entidades introducir estos criterios sin un mercado amplio que pueda responder a esta demanda (Moreno, 2006). En segundo lugar, hasta ahora no es legalmente obligatorio para las entidades la introducción de criterios ambientales en sus contrataciones. A pesar de que en el año 2012 se promovió desde la Cámara de Representantes el proyecto de Ley 237 para fomentar los criterios ambientales en las adquisiciones públicas; este proyecto no logró convertirse en ley debido a que fue archivado por cambio de legislatura (Matera, 2018). En tercer lugar, el desarrollo de la contratación pública sostenible no ha tenido una implementación significativa en las entidades estatales, pues, según el informe de la Organización para la Cooperación y el Desarrollo Económicos (OCDE), la contratación verde se está implementando en "algunas" de las entidades públicas (OCDE, 2020). Asimismo, debido a que el Plan de Acción Nacional de Compras Públicas Sostenibles se estableció con un horizonte a 2020, aún no se cuenta con una evaluación que dé cuenta de los resultados en el cumplimiento de sus objetivos y determinar si sus acciones fueron suficientes para implementar las compras públicas sostenibles en Colombia y si se lograron cambios en los patrones de producción y consumo que consideren el ciclo de vida, en línea con lo planteado en ODS número 12. 
No obstante, a decir por el avance general en el cumplimiento de los ODS para Latinoamérica y el Caribe, el panorama no resulta alentador, toda vez que:

En términos generales, la mayoría de países de la región evidencia un avance en el cumplimiento de los ODS desde su adopción en 2015. Sin embargo, este resulta insuficiente para el cumplimiento de los objetivos trazados a 2030. En promedio, los países mejoraron en 0.67 puntos en el Índice ODS 2019, lo que representa un avance de 1.09 puntos porcentuales con respecto a la línea de base. Si mantenemos este ritmo de avance anual, el cumplimiento de los 17 Objetivos en los 24 países de la región tomaría más de 50 años. Esto evidencia la magnitud del rezago al cual nos enfrentamos y resalta la urgencia de acelerar esfuerzos, si realmente esperamos cumplir con los ODS a 2030 (Centro de los Objetivos de Desarrollo Sostenible para América Latina y el Caribe, 2020, p. 21).

Así las cosas, a pesar de que las licencias ambientales y la contratación pública sostenible son los elementos predilectos con los que se puede aplicar plenamente la dimensión ambiental del desarrollo sostenible en el contrato de obra pública, lo estudiado en la presente sección permitió identificar que no se ha podido aprovechar al máximo estas herramientas de gestión ambiental para articular dicha dimensión en este tipo de contratos, pues aunque ambos instrumentos cuentan con un amplísimo respaldo constitucional, legal y de política pública (al menos en el caso de la contratación sostenible), hoy en día no cumplen plenamente los propósitos para los que fueron establecidos.

En el caso de las licencias ambientales, aunque cuenta paradógicamente con un extensa producción normativa, se ha evidenciado un proceso de des-regularización o flexibilización de las actividades sujetas a licenciamiento sin una justificación técnica, se ha tendido a acotar los términos para su expedición, y se presentan serias falencias en la calidad del estudio y trámite de la licencia, en los procesos de 
participación ciudadana, consultas previas y en el seguimiento posterior del cumplimiento de las actividades autorizadas, estas falencias, a su vez, están asociadas con un debilitamiento de la institucionalidad ambiental, con lo cual se ha terminado por desfigurar el propósito principal de las licencias para la evaluación, planificación ambiental y toma de decisiones, reduciéndola a un mero trámite administrativo.

Esta situación resulta preocupante teniendo en cuenta que la construcción de obras públicas es una de las actividades que más impactos genera al ambiente, por lo que no se cuenta con un instrumento sólido para poder administrar de manera adecuada estos impactos, y al no contar con ello, también se debilita el principio de planeación en la contratación de las obras, considerando su aplicabilidad en todas las etapas del contrato, pues se pierde una herramienta fundamental para valorar y gestionar los impactos ambientales que pueden comprometer el cumplimiento del desarrollo sostenible en sus concepción intergeneracional, de servicios ecosistémicos y límites ambientales.

Por su parte la contratación pública sostenible apenas está en un incipiente desarrollo. Si bien cuenta con un respaldo importante en materia de política pública, y el marco jurídico contractual permite la inclusión de consideraciones ambientales en la contratación, no es legalmente obligatorio; no se ha podido implementar de forma significativa dentro de las entidades estatales; además, aún no se conocen los resultados del Plan de Acción Nacional de Compras Públicas Sostenibles, con lo que se podría determinar un balance del todo positivo. Sin embargo, dado que esta es una estrategia para cumplir con el ODS número 12, el nivel avance en general de estos objetivos presenta preocupante lentitud para su consecución a 2030.

Ciertamente, tanto en las licencias como en la contratación sostenible, un marco legal (incluso constitucional) no es suficiente, la voluntad política no ha contribuido lo necesario para implementar 
y consolidar los propósitos de estas dos herramientas, de hecho, la visión cortoplacista del desarrollo y el sentido económico que los gobiernos han dado a los recursos naturales parecen ir en sentido contrario con los postulados del desarrollo sostenible previstos en la constitución, en la ley y en los compromisos internacionales en torno a los ODS, y en el medio están las obras públicas, pues su carácter instrumental para alcanzar el desarrollo y el crecimiento económico, social y ambiental entra en tensión con las diferentes visiones de desarrollo, lo cual degenera en conflictos ambientales.

\section{Conclusiones}

Los contratos de obra pública poseen un carácter estratégico como herramienta de política pública para concretar las metas y los objetivos establecidos en estas, las cuales a la luz de los artículos 2 y 209 de la Constitución Política de 1991 deben orientar sus propósitos al interés general y al cumplimiento de los fines del Estado social y de derecho. Por ello, ese carácter instrumental de los contratos de obra está asociado con los procesos de crecimiento del país y al logro del desarrollo sostenible que consagró el artículo 80 de la Constitución. No obstante, estos contratos también producen altos impactos al ambiente debido al uso de los recursos naturales, consumo energético y generación de contaminación y residuos.

Aunado a lo anterior, el rápido avance en el deterioro de los recursos naturales, producto de la pérdida de diversidad biológica y el cambio climático, está excediendo límites planetarios que ponen en riesgo servicios ecosistémicos necesarios para el funcionamiento del planeta. Esta circunstancia evidencia un atraso de la arista ambiental respecto a la dimensión económica y social del desarrollo sostenible, con lo cual es necesario que el contrato de obra pública haga uso de herramientas que le permitan planificar y gestionar los recursos naturales que requiere y los efectos que produce en su ejecución, potenciando su carácter estratégico para la protección del ambiente. 
En este sentido, de acuerdo con la pregunta de investigación formulada referente a si el carácter instrumental del contrato de obra pública, para el crecimiento social y económico en Colombia, se ha articulado o no con la aplicación del principio de desarrollo sostenible consagrado en la Constitución Política de 1991 y en la Ley 99 de 1993, desde su dimensión ambiental, la respuesta es negativa, por cuanto los principales elementos con los que se puede dar aplicación a dicho principio en su aspecto ambiental, es decir, las licencias ambientales y la contratación pública sostenible, hoy en día no cumplen el propósito para el cual fueron establecidos estos elementos.

Lo anterior se explica porque las licencias ambientales se han convertido en un simple trámite administrativo, como consecuencia de un proceso de flexibilización de las actividades sujetas a licenciamiento, serias falencias en el trámite de expedición y seguimiento, escasa participación ciudadana, así como de una débil institucionalidad de las autoridades ambientales. Respecto a la contratación pública sostenible, pese a un importante respaldo jurídico y de política pública, aún no es obligatorio para las entidades públicas y en todo caso su implementación apenas está en un incipiente desarrollo, lo cual no se compadece con el avance que se requiere de esta herramienta para dar cumplimiento al ODS número 12 para el año 2030.

Estos escenarios permiten evidenciar una falta de la voluntad política para implementar y consolidar los propósitos de planificación y protección ambiental, así como para preveer obligatoriamente consideraciones ambientales en la contratación pública, debido a una visión cortoplacista del desarrollo por parte de los gobiernos de turno, en la que han prevalecido los beneficios económicos en el uso de los recursos naturales por encima de su protección y conservación, pese a los deberes del Estado frente a la garantía del desarrollo sostenible prevista en la Constitución Política de 1991, en la Ley 99 de 1993 y en los compromisos internacionales entorno al cumplimiento de los ODS. 
En consecuencia, estas evidencias permiten aceptar la hipótesis planteada al inicio de la presente investigación. Adicionalmente, acorde con el desarrollo de este escrito, se logró cumplir con el objetivo general que se formuló, pues se logró determinar que el carácter instrumental del contrato de obra pública para el crecimiento social y económico en Colombia no se ha logrado articular con la aplicación del principio de desarrollo sostenible, consagrado en la Constitución Política de 1991 y en la Ley 99 de 1993 desde su dimensión ambiental, tal como se pasa a exponer a continuación.

De acuerdo con los objetivos específicos, en primer lugar, se identificó que el carácter instrumental del contrato de obra pública, a la luz de los fines de la contratación estatal del artículo 3 de la Ley 80 de 1993, se manifiesta que es un elemento estratégico de las políticas públicas, desde el enfoque de la nueva gestión pública, porque, al ser un contrato estatal, en él se materializan los objetivos establecidos en dichas políticas, las cuales se crean para resolver problemáticas concretas, satisfacer intereses comunes y garantizar condiciones de bienestar a los asociados, quienes participan en la contratación como destinatarios y como colaboradores en el cumplimiento de los propósitos que se buscan con las políticas y esta interacción imprime dinamismo a los procesos de crecimiento económico y social del país.

En segundo lugar, se pudo establecer que, en el marco del desarrollo sostenible, consagrado en la Constitución Política de 1991, la Ley 99 de 1993 y la conceptualización que ha enriquecido su contenido, existen dos elementos principales que posibilitan la plena aplicación de la dimensión ambiental de este principio en el contrato de obra pública, que son la licencia ambiental y la contratación pública sostenible. El primero, como herramienta fundamental para la planificación ambiental y toma de decisiones, directamente asociado con los proceso de planeación en los procesos de contratación, asimismo, permite valorar los impactos ambientales y establecer me- 
didas de prevención, protección, manejo y compensación de tales impactos. El segundo, como estrategia para lograr el cumplimiento del ODS número 12, proporciona a las entidades estatales de bases conceptuales y metodológicas para incluir criterios de sostenibilidad ambiental en sus contrataciones. Dentro del contrato de obra cobra gran importancia, dado que la implementación de características técnicas ambientalmente sostenibles en los insumos o procesos de producción o construcción de obras resulta fundamental para mejorar la eficiencia en el uso o explotación de los recursos naturales al considerar los ciclos de vida de tales insumos o procesos, que abarcan incluso hasta la disposición final de los mismos y, de esta manera, generar cambios en los patrones de producción y consumo sostenible en las compras públicas.

Por último, se identificaron los aspectos que han imposibilitado la articulación del principio de desarrollo sostenible, desde su dimensión ambiental en el contrato de obra pública. Estos aspectos tienen que ver con el debilitamiento del propósito las licencias como consecuencia de proceso de desregularización o flexibilización de las actividades sujetas a licenciamiento, sin una justificación técnica, la tendencia a reducir los términos para su expedición, las falencias en la calidad del estudio y trámite de la licencia, en los procesos de participación ciudadana, consultas previas y en el seguimiento posterior del cumplimiento de las actividades autorizadas, aunado al declive de la institucionalidad de las autoridades, ha mermado su rigurosidad como herramienta de evaluación de impacto ambiental.

A su vez, la contratación pública sostenible no se ha podido consolidar dentro de las entidades estatales, debido a que la introducción de criterios ambientales en el proceso de contratación hoy por hoy no es legalmente obligatorio, y por eso solo se ha implementado en algunas de las entidades. Además, no se cuenta aún con resultados del Plan de Acción Nacional de Compras Públicas Sostenibles, con lo que se podrían determinar logros en los objetivos propuestos 
para dicho plan para contribuir al cumplimiento del ODS número 12; sin embargo, el nivel de avance en general de los ODS presenta una preocupante lentitud para su consecución a 2030.

\section{Referencias}

Acevedo, H., Ramírez, D. y Vásquez, A. (2012). Sostenibilidad: Actualidad y necesidad en el sector de la construcción en Colombia. Revista Gestión y Ambiente, 15(1), 105-117.

Amaya, 0. (2016). La Constitución Ecológica de Colombia. Bogotá: Universidad Externado de Colombia.

Aponte, I. (2014). Las fallas de planeacion y su incidencia en el contrato estatal de obra. Revista digital de derecho administrativo, (11), 177-207. https://dialnet.unirioja.es/ servlet/oaiart?codigo $=5137180$

Araújo, R. (2011). Delimitación conceptual de la participación ciudadana administrativa. En: R. Araujo y M. Torres (Ed.), Retos de la democracia y de la participación ciudadana (pp. 85-116). Bogotá: Editorial Universidad del Rosario.

Banco de la República. (2019). Informe de la junta directiva al Congreso de la República. https://repositorio.banrep.gov.co/bitstream/handle/20.500.12134/9732/informecongreso-julio-2019.pdf?sequence $=8$

Briceño, A. (2008). Introducción al sello ambiental en Colombia. El sello Ambiental Colombiano como modelo de etiqueta para un Estado en desarrollo. En Universidad Externado de Colombia. (Ed.), Lecturas sobre derecho del medio ambiente. Tomo VIII (pp. 43-98). Bogotá: Universidad Externado de Colombia.

Centro de los Objetivos de Desarrollo Sostenible para América Latina y el Caribe. (2020). Índice ODS 2019 para América Latina y el Caribe. https://cods.uniandes.edu.co/wpcontent/uploads/2020/06/Índice-ODS-2019-para-América-Latina-y-el-Caribe-2.pdf

Colombia Compra Eficiente. (s.f.). Guía de compras públicas sostenibles con el ambiente. https://www.colombiacompra.gov.co/sites/cce_public/files/cce_documents/cce_ guia_cp_sostenibles.pdf

Contraloría General de la República. (2017). El proceso administrativo de licenciamiento ambiental en Colombia. https://www.contraloria.gov.co/documents/20181/465175/ $\mathrm{El}+$ proceso + administrativo $+\mathrm{de}+$ licenciamiento + ambiental $+e n+$ Colomb ia $+2017 . p d f$ 
Díaz, F., Murcia, J., Onate, G., Rodríguez, S., Rodríguez, G., Rojas, R. y Santana, L. (2019). Proyectos Formulación y criterios de evaluación. Bogotá: Alfaomega.

Gómez, L. (2009). Técnica, ambiente y sociedad del riesgo. Revista Gestión y Ambiente, 12(2), 7-15.

Gómez, R., Monroy, J. y Rodríguez, G. (2013). Las licencias ambientales en Colombia. Bogotá: Editorial Ibañez.

Graglia, J. (2016). Modelo relacional de políticas públicas. En: D. Calderón (Compilador), Políticas públicas: retos y desafíos para la gobernabilidad (pp. 37-58). Bogotá: Universidad Santo Tomás.

Guhl, E. (2015). Evolución del ministerio de ambiente de Colombia en sus primeros veinte años: 1994-2014. En: E. Guhl y P. Leyva, La gestión ambiental en Colombia, 19942014: ¿un esfuerzo insostenible? (pp. 25-113). Bogotá: Foro Nacional Ambiental.

Matera, M. (2018). Compras públicas sostenibles. Análisis de la inclusión de criterios de sostenibilidad en los acuerdos marco de precios celebrados por la agencia nacional de contratación "Colombia Compra Eficiente" [tesis de maestría no publicada]. Repositorio Universidad Santo Tomás. https://repository.usta.edu.co/bitstream/handle/1 1634/16010/2019marcelamatera.pdf? sequence $=5$ \&isAllowed $=y$

Mance, H. (2008). La política de la sostenibilidad: Ascenso y Declive del Ministerio del Medio Ambiente Colombiano. En M. Rodríguez (Ed.), Gobernabilidad, Instituciones y Medio Ambiente en Colombia (pp. 101-251). Bogotá: Foro Nacional Ambiental.

Ministerio de Ambiente y Desarrollo Sostenible. (s.f.). Compras públicas sostenibles. Consultado el 17 de octubre de 2020. https://www.minambiente.gov.co/index.php/asuntos-ambientales-sectorial-y-urbana/sostenibilidad-sectores-productivos/compraspublicas-sostenibles

Ministerio de Ambiente y Desarrollo Sostenible. (s. f.). Sello Ambiental Colombiano. Consultado el 17 de octubre de 2020. https://www.minambiente.gov.co/index.php/asuntos-ambientales-sectorial-y-urbana/sostenibilidad-sectores-productivos/sello-ambiental-colombiano

Ministerio de Ambiente y Desarrollo Sostenible. (2017). Plan de Accion Nacional de Compras Publicas Sostenibles 2016-2020. https://www.minambiente.gov.co/images/ AsuntosambientalesySectorialyUrbana/pdf/compras_públicas/Plan_de_Accion_Nacional_de_Compras_Publicas_Sostenibles_2016-2020.pdf

Ministerio de Ambiente, Vivienda y Desarrollo Territorial. (2010). Fortalecimiento de las capacidades para las compras públicas sostenibles en Colombia. 
Moreno, H. (2006). Contratación sostenible. En: G. Herrera, B. Londoño y G. Rodríguez (Ed.), Perspectivas del derecho ambiental en Colombia (pp. 390-408). Bogotá: Editorial Universidad del Rosario.

Munévar, C. y Valencia, J. (2020). Los conflictos socio-ambientales en Colombia en el contexto de las Licencias Ambientales y el acceso a la justicia. Revista Jurídicas, 17(1), 42-63.

Naciones Unidas. (s.f.a). Declaración de Rio sobre el Medio Ambiente y el Desarrollo. https://www.un.org/spanish/esa/sustdev/agenda21/riodeclaration.htm

Naciones Unidas. (s.f.b). Objetivos de desarrollo sostenible. https://www.un.org/sustainabledevelopment/es/

Organización para la Cooperación y el Desarrollo Económicos. (2020). Panorama de las Administraciones Públicas América Latina y el Caribe 2020. https://www.oecd.org/ gov/gov-at-a-glance-alc-country-factsheet-es-2020-colombia.pdf

Peña, V. (2016). El sinuoso camino hacia un Estado de políticas públicas. En: D. Calderón (Compilador), Políticas públicas: retos y desafíos para la gobernabilidad (pp. 17-36). Bogotá: Universidad Santo Tomás.

Procolombia. (s. f.). Inversión en el sector materiales de construcción en Colombia. Consultado el 12 de septiembre de 2020. https://www.inviertaencolombia.com.co/sectores/ manufacturas/materiales-de-construccion.html

Rezzoagli, B. (2016). Contratación pública para objetivos y resultados: aproximación y discusión teórica. Revista De Derecho Público, (37), 1-23. https://doi.org/10.15425/ redepub.37.2016.01

Rodríguez, B. (2018). El desarrollo sostenible en los albores de los ODS y del Acuerdo de París. En: B. Rodríguez y M. Vélez (Ed.), Gobernanza y gerencia del desarrollo sostenible (pp. 3-35). Bogotá: Universidad de los Andes. https://elibro.net/es/ereader/ usta/118317?page $=37$

Rodríguez, G. (2012). La consagración de los derechos ambientales en las constituciones políticas de Colombia, Ecuador y Bolivia. En: I. Páez y G. Rodríguez (Ed.), Temas de derecho ambiental: una mirada desde lo público (pp. 1-53). Bogotá: Editorial Universidad del Rosario.

Sachs, J. (2015). La era del desarrollo sostenible. Barcelona: Ediciones Deusto

Santa María, M. (2020, 15 de abril). Desempeño reciente del sector construcción y perspectivas 2020. La República. https://www.larepublica.co/analisis/anif-2941063/ desempeno-del-sector-construccion-y-perspectivas-2020-2991939 
Sistema económico Latinoamericano y del Caribe. (2014). Las compras públicas como herramienta de desarrollo en América Latina y el Caribe. Caracas: http://www.sela.org/ es/centro-de-documentacion/base-de-datos-documental/bdd/28495/las-compraspublicas-como-herramienta-de-desarrollo-en-america-latina-y-el-caribe-1

Sleman, J. y Velásquez, C. (2016). La licencia ambiental: ¿instrumento de comando y control por excepción? Vniversitas, 132, 483-514. 
